PROCEEDINGS OF THE

AMERICAN MATHEMATICAL SOCIETY

Volume 128, Number 3, Pages 877-884

$\mathrm{S}$ 0002-9939(99)05179-5

Article electronically published on July 6, 1999

\title{
ON DIOPHANTINE SETS OVER POLYNOMIAL RINGS
}

\author{
KARIM ZAHIDI
}

(Communicated by Carl G. Jockusch, Jr.)

\begin{abstract}
We prove that the recursively enumerable relations over a polynomial ring $R[t]$, where $R$ is the ring of integers in a totally real number field, are exactly the Diophantine relations over $R[t]$.
\end{abstract}

\section{INTRODUCTION}

Since the characterisation of recursively enumerable relations over the ring of rational integers $\mathbf{Z}$ as Diophantine relations (see [9]), various authors have been interested in extending this result to other rings. The first rings for which this problem was considered are the rings of algebraic integers in finite extensions of the field of rational numbers. The original result extends to several classes of algebraic number rings but the problem for an arbitrary algebraic number ring remains open. To prove that the recursively enumerable relations are exactly the Diophantine relations it clearly suffices to give a Diophantine definition of the ring of rational integers into such a ring of algebraic integers. For more details the reader may consult [2], [4], [7], [11], [14], [15].

We briefly recall the relevant definitions concerning Diophantine and recursively enumerable relations.Let $R$ be a commutative domain. We say that an $n$-ary relation $D$ over $R$ is Diophantine if there exists a polynomial $P\left(x_{1}, \cdots, x_{n}, y_{1}, \cdots, y_{m}\right)$ $\in R[\bar{X}, \bar{Y}]\left(\bar{X}=\left(x_{1}, \cdots, x_{n}\right), \bar{Y}=\left(y_{1}, \cdots, y_{m}\right)\right)$ such that

$$
D(\bar{X}) \leftrightarrow \exists \bar{Y} \in R^{m}: P(\bar{X}, \bar{Y})=0 .
$$

It's well known that the disjunction of two Diophantine relations is again a Diophantine relation and in case the fraction field of $R$ is not algebraically closed, the same holds true for a conjunction of Diophantine relations.Let $R$ be a ring. Then we say that $R$ is a recursive ring if there exists a map $\theta$ from $\mathbf{N}$ to $R$ such that $\theta$ is onto and such that $\theta^{-1}(R)$ is a recursively enumerable subset of $\mathbf{N}$ and such that the functions

$$
A: \theta^{-1}(R) \times \theta^{-1}(R) \rightarrow \theta^{-1}(R):(n, m) \rightarrow \theta^{-1}[\theta(n)+\theta(m)]
$$

and

$$
M: \theta^{-1}(R) \times \theta^{-1}(R) \rightarrow \theta^{-1}(R):(n, m) \rightarrow \theta^{-1}[\theta(n) \theta(m)]
$$

Received by the editors September 16, 1997 and, in revised form, April 22, 1998.

1991 Mathematics Subject Classification. Primary 03D20; Secondary 11U05, 12L05.

The author would like to thank Professor J. Van Geel for his help during the preparation of this work.

(C)1999 American Mathematical Society 
are recursive functions on $\theta^{-1}(R)$. The map $\theta$ is called an admissible indexing of the ring $R$. Given an indexing $\theta$ of a recursive ring $R$, the notions of $\theta$-recursive function, $\theta$-recursive relation, $\theta$-recursively enumerable relation can easily be defined (in the sequel we will drop the explicit reference to the indexing). For example an $n$-ary relation $\mathcal{F}$ is called recursively enumerable if the relation $\overline{\mathcal{F}}$ defined as

$$
\overline{\mathcal{F}}\left(k_{1}, \cdots, k_{n}\right) \leftrightarrow \exists X_{1}, \cdots, X_{n} \in R: \mathcal{F}\left(X_{1}, \cdots, X_{n}\right) \wedge X_{i}=\theta\left(k_{i}\right)
$$

is a recursively enumerable relation on $\theta^{-1}(R)$ (and hence on the natural numbers). If the ring under consideration is a polynomial ring $R[t]$, for a natural number $n$, $n \in \theta^{-1}(R[t])$, we will denote $\theta(n)$ by $P_{n}$ and call $n$ the index of the polynomial $P_{n}$. We call $\theta$ an effective indexing if and only if the following condition holds: For every polynomial $F$, given the index $n$ of $F$ (i.e. $P_{n}=F$ ) one can effectively calculate how many non-zero coefficients $F$ has and one can effectively calculate the indices of these coefficients. In the sequel we will always assume that the indexing under discussion is effective in this sense (it is clear that such an indexing always exists whenever $R$ is a recursive ring). For more information about recursiveness over arbitrary algebraic structures the reader may consult [13].

In [8] Davis and Putnam proved that every recursively enumerable set over $\mathbf{Z}$ is Diophantine over the polynomial ring in one variable over $\mathbf{Z}, \mathbf{Z}[t]$. Later Denef used this result in [3] to prove that any recursively enumerable relation over $\mathbf{Z}[t]$ is Diophantine over $\mathbf{Z}[t]$. Using similar techniques as in [3] we obtain the following generalisation of Denef's result:

Theorem. Let $R$ be the ring of integers in a totally real number field $K$ (i.e. $K$ has only real embeddings into the field of complex numbers $\mathbf{C})$. Let $\theta$ be an effective indexing of $R[t]$; then every $\theta$-recursively enumerable relation over $R[t]$ is a Diophantine relation over $R[t]$.

Under the assumption that the ring of rational integers is a Diophantine subset of $R[t]$ it clearly suffices to obtain a Diophantine definition of the indexing. Indeed if the relation $n \in \theta^{-1}(R[t]) \wedge F=P_{n}$ is Diophantine over $R[t]$, then for any recursively enumerable relation $\mathcal{F}$ we have the following equivalence:

$$
\begin{aligned}
\mathcal{F}\left(X_{1}, \cdots, X_{n}\right) \leftrightarrow & \exists l_{1}, \cdots, l_{n} \in R[t]: \\
& \wedge\left(\bigwedge_{i=1}^{n} l_{i} \in \theta^{-1}(R[t])\right) \\
& \wedge\left(\bigwedge_{i=1}^{n} X_{i}=P_{l_{i}}\right) \\
& \wedge \overline{\mathcal{F}}\left(l_{1}, \cdots, l_{n}\right) .
\end{aligned}
$$

Now since $\overline{\mathcal{F}}$ is by definition a recursively enumerable relation over $\mathbf{N}$, and $\theta^{-1}(R[t])$ is a recursively enumerable subset of $\mathbf{N}$, they are both Diophantine over $\mathbf{Z}$ and thus by assumption also Diophantine over $R[t]$ and hence the right-hand side of the equivalence is Diophantine.

In the first section we give a Diophantine definition of the ring of algebraic integers $R$ of a real number field $K$ in the polynomial ring $R[t]$. From this we obtain a Diophantine definition of $\mathbf{Z}$ in $R[t]$ if we know that $\mathbf{Z}$ is a Diophantine subset of $R$. This was conjectured by Denef and Lipshitz in [7]. The conjecture has been proven for several classes of number fields including the class of quadratic number fields (see [3]) and more generally abelian number fields (see [15]), the class of totally real number fields (see [4]) and the class of number fields with exactly two nonreal embeddings (see [11] and [14]). The Main Lemma of Section 3 proves that the indexing is Diophantine in case the number field is totally real. 
Throughout the first two sections of this paper $K$ will denote a real algebraic number field of degree $r, R$ will denote its ring of algebraic integers and the elements $\alpha_{1}, \cdots, \alpha_{r} \in K$ will denote an integral basis for $R$. By $\sigma$ (or $\sigma_{i}$ ) we will always denote a real embedding of $K$ into the field of complex numbers. In the third section $K$ will denote a totally real number field.

\section{Diophantine Definition of the Constants}

We start by defining, in a Diophantine way, a set of constants which contains elements with arbitrary large absolute value with respect to some real embedding $\sigma$ (Lemma 1.1). This together with the fact that given an algebraic integer $r$ and a non-constant polynomial $F \in R[t]$, which takes only positive values (w.r.t. $\sigma$ ), there is always a value $t_{0} \in R$ such that $\sigma\left(F\left(t_{0}\right)\right)>\sigma(r)$ will give a Diophantine definition of the constants (Lemma 1.3) (a similar technique was used in [8] to prove that $\mathbf{Z}$ is a Diophantine subset of $\mathbf{Z}[t])$.

Lemma 1.1. Let $d$ be a natural number, such that $d$ is not a square in $K$. The equation

$$
X^{2}-d Y^{2}=1
$$

has only constant solutions in $R[t]$. For every natural number $n$ we can find a solution $(X, Y)$ of $(*)$, with $X, Y \in \mathbf{Z}$ such that $n<|Y|$.

Proof. Suppose there are non-constant polynomials $f$ and $g$ that satisfy $(*)$. Certainly $f$ and $g$ will have the same degree, say $\operatorname{deg}(f)=\operatorname{deg}(g)=k$. Now if $f$ and $g$ satisfy equation $(*)$, then we must have the following relation between the highest degree coefficients:

$$
a_{k}^{2}-d b_{k}^{2}=0
$$

(where $a_{k} \neq 0$ and $b_{k} \neq 0$ are the highest degree coefficients of $f$ and $g$ ). This implies that $d$ is a square in $K$.

Note that $X^{2}-d Y^{2}$ is the norm form of the real quadratic extension $\mathbf{Q}(\sqrt{d})$. By Dirichlet's Unit Theorem, there are infinitely many units, so equation $(*)$ has infinitely many integer solutions. This readily implies that there are solutions in $\mathbf{Z}$ with arbitrarily large values of $Y$.

From now on we assume that $d$ is a natural number such that $d$ is not a square in $K$. The following lemma, first used in [4], gives a characterisation of the elements $x \in R$ for which $\sigma(x) \geq 0$ for some real embedding $\sigma$ of $K$.

Lemma 1.2. Let $K$ be a real number field and let $\sigma$ be a real embedding of $K$ and suppose that $c \in R$ satisfies $\sigma(c)>0$ and $\sigma_{j}(c)<0$ for all real embeddings $\sigma_{j} \neq \sigma$. Then we have the following equivalence:

$$
\sigma(x) \geq 0 \leftrightarrow \exists x_{0}, \cdots x_{4} \in R: x_{0} \neq 0 \wedge x_{0}^{2} x=x_{1}^{2}+\cdots+c x_{4}^{2} .
$$

The proof of this lemma is an application of the Hasse-Minkowski theorem on quadratic forms (for the proof the reader may consult [4]).

So from now on fix a real embedding $\sigma$ and a constant $c$ which satisfy the conditions of Lemma 1.2.

To give a Diophantine definition of the constants in $R[t]$ it suffices to give a Diophantine definition of the positive (with respect to a fixed real embedding $\sigma$ ) constants, since

$$
x \in R \leftrightarrow(x \in R \wedge \sigma(x) \geq 0) \vee(x \in R \wedge \sigma(-x) \geq 0) .
$$


In order to give the required Diophantine definition, we first need to show that the condition $x_{0} \neq 0$ is Diophantine over $R[t]$. In [7] it was shown that $x_{0} \neq 0$ is Diophantine over $R$. We will use a similar trick to construct a Diophantine subset $V \in R[t]$, such that every element of $V$ is non-zero and such that $R \backslash\{0\}$ is a subset of $\mathrm{V}$.

Lemma 1.3. Let $V$ be the subset of $R[t]$ defined by:

$$
x \in V \leftrightarrow \exists a, b \in R[t]: a x=(2 b-1)(3 b-1) .
$$

Then every non-zero element of $R$ is an element of $V$. Furthermore every element of $V$ is non-zero.

Proof. Suppose $x$ is a non-zero element in $R$; we have to show that the right-hand side can be satisfied. Let $y=N_{\mathbf{Q}(x) / \mathbf{Q}}(x)\left(N_{\mathbf{Q}(x) / \mathbf{Q}}\right.$ denotes the norm of the field extension $\mathbf{Q}(x) / \mathbf{Q})$; then $y \in \mathbf{Z}$ and $y \neq 0$. Write $y$ as $2^{n} y^{\prime}$ with $y^{\prime}$ an odd integer. Then by the Chinese Remainder Theorem we can find an integer $b$ such that $2 b-1$ is divisible by $y^{\prime}$ and $3 b-1$ is divisible by $2^{n}$. Hence $q=\frac{(2 b-1)(3 b-1)}{y}$ is an integer. Now clearly $a=q \frac{y}{x}$ is in $R$. With these choices of $a$ and $b$ the right-hand side will be satisfied.

Suppose $x=0$ satisfies the right-hand side of the equivalence. This implies that either $a=\frac{1}{2}$ nor $b=\frac{1}{3}$, but then neither $a$ nor $b$ is in $R$.

Lemma 1.4. For any $x$ in $R[t]$ we have:

$$
\begin{aligned}
& x \in R \wedge \sigma(x) \geq 0 \leftrightarrow \exists z, w, x_{o}, \cdots, x_{9} \in R[t]: \\
& x_{0} \in V \wedge x_{5} \in V \\
& x_{0}^{2} x=x_{1}^{2}+\cdots+c x_{4}^{2} \\
& \wedge z^{2}-d w^{2}=1 \\
& \wedge x_{5}^{2}(w-x)=x_{6}^{2}+\cdots+c x_{9}^{2} .
\end{aligned}
$$

Proof. First suppose that $x$ is a constant such that $\sigma(x) \geq 0$. Then by Lemma 1.2 and Lemma 1.3 we can find constants $x_{0}, \cdots, x_{4}$ such that $(*)$ and $(* *)$ are satisfied. Now choose integers $z$ and $w$ which satisfy $(* * *)$ and so that $w \geq \sigma(x)$ (this is always possible by Lemma 1.1). Then obviously it is the case that $\sigma(w-x) \geq 0$ and hence by Lemma $1.2(* * * *)$ can be satisfied.

Conversely suppose that the right-hand side is satisfied for some non-constant element $x$ in $R[t]$. Conditions $(*)$ and $(* *)$ force at least one of the $x_{i}$ 's to be non-constant; hence $x$ is a polynomial of even degree and it takes on arbitrarily large values (with respect to $\sigma$ ) as $t$ ranges over $K$. Now by condition $(* * *) w$ is some fixed constant in $R$ so we can find $\alpha$ in $R$ such that $\sigma(w-x(\alpha))<0$, which contradicts $(* * * *)$, so we can conclude that $x$ must be a constant.

\section{Some Diophantine Definitions}

From now on we assume that $K$ is a real number field for which the conjecture of Denef and Lipshitz holds. By the results of the previous section we can assume that $\mathbf{Z}$ is a Diophantine subset of the ring of integers $R$ of $K$.

Lemma 2.1. The relation $\bigwedge_{i=1}^{r} v_{i} \in \mathbf{Z} \wedge F \in R[t] \wedge x \in \mathbf{Z} \wedge F(x)=v_{1} \alpha_{1}+\cdots+$ $v_{r} \alpha_{r}$ in the variables $x, v_{i}, F$ is Diophantine over $R[t]$. 
Proof. The relation is equivalent to

$\exists G, h \in R[t]:$

$h \in R \wedge F-h=(t-x) G \wedge x \in \mathbf{Z} \wedge v_{1}, \cdots, v_{r} \in \mathbf{Z} \wedge h=v_{1} \alpha_{1}+\cdots+v_{r} \alpha_{r}$.

Since both $R$ and $\mathbf{Z}$ are Diophantinely definable over $R[t]$ we get the Diophantine definition.

In the next section we will make use of the solutions of the following Pellequation:

$$
X^{2}-\left(t^{2}-1\right) Y^{2}=1 .
$$

The solutions of this equation satisfy a lot of interesting properties and were first studied (as an equation over $\mathbf{Z}[t]$ ) by Davis and Putnam (in [8]) and later by Denef (over arbitrary domains - see [5] and [6] ). We will briefly discuss the properties we need without proofs. The reader can check the proofs in any of the above cited references.

Since $t^{2}-1$ is not a square in $R[t]$, the relation

$$
X_{n}+\sqrt{t^{2}-1} Y_{n}=\left(t+\sqrt{t^{2}-1}\right)^{n}
$$

defines uniquely for every natural number $n$ the polynomials $X_{n}, Y_{n}$ in $\mathbf{Z}[t] \subset R[t]$.

One checks (see [5]) that every pair $\left(X_{n}, Y_{n}\right)$ is a solution of the Pell-equation $(*)$ and conversely if $(X, Y)$ satisfies the Pell equation $(*)$, then, for some natural number $n,(X, Y)=\left( \pm X_{n}, \pm Y_{n}\right)$. The following two facts can easily be proved using the binomial theorem:

$$
\operatorname{deg} Y_{n}=n-1 \text { and } Y_{n}(1)=n .
$$

Lemma 2.2. The relation $n \in \mathbf{N} \wedge F \in R[t] \wedge F=Y_{n}$ in the variables $F$ and $n$ is Diophantine over $R[t]$.

Proof. By the above stated properties of the polynomials $X_{n}$ and $Y_{n}$ the relation $n \in \mathbf{N} \wedge F=Y_{n}$ is equivalent to:

$$
\exists X \in R[t]: X^{2}-\left(t^{2}-1\right) F^{2}=1 \wedge F(1)=n \wedge n \in \mathbf{N} .
$$

Now by Lagrange's theorem $\mathbf{N}$ is a Diophantine subset of $\mathbf{Z}$ and hence of $R[t]$. This together with Lemma 2.1 proves that the right-hand side is Diophantine.

Definition 2.3. Let $F$ be a polynomial in $R[t]$; we say that $F$ is positive-definite on $K$ (notation $\operatorname{Pos}(F)$ ) if and only if $\sigma(F(t)) \geq 0, \forall t \in K$ and for all real embeddings $\sigma$.

Lemma 2.4. The relation Pos is Diophantine over $R[t]$.

Proof. We claim that the following equivalence holds:

$$
\operatorname{Pos}(F) \leftrightarrow \exists F_{1}, \cdots, F_{5}, c \in R[t]: c \in V \wedge c^{2} F=F_{1}^{2}+\cdots+F_{5}^{2} .
$$

Suppose a polynomial satisfies the right-hand side of the equivalence; then clearly $F$ satisfies Pos.

Conversely suppose that $F$ is positive definite on $K$. Then it follows from a theorem of Pourchet (see [10], Theoreme 2; see also [1], Proposition 1.3) that $F$ can be written as a sum of five squares in $K[t]$. Now multiplying by a suitable non-zero constant $c^{2}$ these polynomials can be taken to be in $R[t]$, and hence the right-hand side is satisfied. 


\section{Main Lemma: Diophantine Definition of the indexing}

From now on we assume that $K$ is a totally real number field.

Lemma 3.1. Let $d$ be a natural number and $F$ a polynomial in $R[t]$. Then the relation $\operatorname{deg} F=d$ is a recursive relation.

Proof. Given a polynomial $F$ and a natural number $d$ we can use the following algorithm to test whether the relation holds:

1. from the index $n$ of $F$ calculate the number of non-zero coefficients of $F$ and their indices;

2. calculate the coefficients and make a list (finite) of all polynomials with the given coefficients and of degree $d$;

3. calculate the indices of each of these polynomials;

4. if the index of $F$ appears on the list, then $F$ has degree $d$; otherwise not.

Lemma 3.2. Let $\mathcal{F}$ be an $n$-ary Diophantine relation on $R[t]$; then $\mathcal{F}$ is a recursively enumerable relation on $R[t]$.

Proof. By assumption there exists a polynomial $P$ with coefficients in $R[t]$ such that:

$$
\mathcal{F}\left(F_{1}, \cdots, F_{n}\right) \leftrightarrow \exists Y_{1}, \cdots, Y_{m} \in R[t]: P\left(F_{1}, \cdots, F_{n}, Y_{1}, \cdots, Y_{m}\right)=0 .
$$

The algorithm consists of listing all $n+m$-tuples over $R[t]$, and testing for each $n+m$ tuple whether it is a zero of the polynomial $P$. This implies that the relation $\overline{\mathcal{F}}$ defined on the indices of the polynomials is a recursively enumerable relation on the natural numbers and hence $\mathcal{F}$ is recursively enumerable.

Definition 3.3. Define the relation $\operatorname{Par}\left(n, b, c, d, v_{1}, \cdots, v_{r}\right)$ on the rational integers as follows:

$\wedge d=\operatorname{deg} P_{n}$

(2) $\wedge \operatorname{Pos}\left(Y_{d+2}^{2}+c-P_{n}^{2}-1\right)$

(3) $\wedge \forall x \in \mathbf{Z}: 0 \leq x \leq d, \quad Y_{d+2}^{2}(x)<b$

(4) $\wedge P_{n}(2 b+2 c+d)=v_{1} \alpha_{1}+\cdots+v_{r} \alpha_{r}$.

Note that Par is indeed a recursively enumerable relation (by Lemmas 3.1 and 3.2 , since Pos is a Diophantine relation over $R[t])$.

Main Lemma. 1. $n \in \theta^{-1}(R[t]) \wedge F=P_{n} \leftrightarrow \exists b, c, d, v_{1}, \cdots v_{r} \in R[t]$ :

$$
\begin{aligned}
& \operatorname{Par}\left(n, b, c, d, v_{1}, \cdots, v_{r}\right) \\
& \wedge \operatorname{Pos}\left(Y_{d+2}^{2}+c-F^{2}-1\right) \\
& \wedge F(2 b+2 c+d)=v_{1} \alpha_{1}+\cdots v_{r} \alpha_{r} .
\end{aligned}
$$

2. The indexing $n \in \theta^{-1}(R[t]) \wedge F=P_{n}$ is Diophantine over $R[t]$.

Proof. 1. Suppose $F=P_{n}$ for some natural number $n$. Then one can easily find natural numbers $b, c$ and rational integers $v_{1}, \cdots, v_{r}$ such that the relation Par and hence the right-hand side are satisfied $\left((2)\right.$ can be satisfied because $\operatorname{deg} P_{n}<$ $\left.\operatorname{deg} Y_{d+2}\right)$. 
Conversely suppose that the right-hand side is satisfied for some natural numbers $c, d, n, b$ and integers $v_{1}, \cdots, v_{r}$; then we have to prove that $F=P_{n}$. From conditions (4) and (7) it follows that

$$
\left(F-P_{n}\right)(2 b+2 c+d)=0
$$

so, if $F \neq P_{n}$, there is some $S \in R[t] \backslash\{0\}$ such that $F-P_{n}=(2 b+2 c+d-t) S(t)$. Now by condition (6), $F$ has degree at most $d+1$, while $P_{n}$ has degree $d$ (by condition (1)); hence $S$ has degree at most $d$. So for some integer $k, 0 \leq k \leq d$, we have $S(k) \neq 0$. Now for at least one real embedding $\sigma$ we have

$$
\left|\sigma\left(\left(F-P_{n}\right)(k)\right)\right|=|(2 b+2 c+d-k)||\sigma(S(k))| \geq 2 b+2 c
$$

(since $k \leq d$ and the fact that given an algebraic integer $a$ in a totally real number field, $a \neq 0$, there is at least one real embedding such that $|\sigma(a)| \geq 1$ ).

On the other hand for any real embedding $\sigma$ we have, for all integers $x, 0 \leq x \leq d$ :

$$
|\sigma(F(x))| \leq\left|\sigma\left(F(x)^{2}+1\right)\right| \leq Y_{d+2}^{2}(x)+c<b+c
$$

and

$$
\left|\sigma\left(P_{n}(x)\right)\right| \leq \sigma\left(P_{n}^{2}(x)+1\right) \leq Y_{d+2}^{2}(x)+c<b+c
$$

(by conditions (2) and (3)), and hence

$$
\left|\sigma\left(\left(F-P_{n}\right)(x)\right)\right|<2 b+2 c
$$

which gives a contradiction.

2. Since the relation $P a r$ is a recursively enumerable relation over $\mathbf{Z}$, it is also Diophantine over $\mathbf{Z}$. Now the result of Section 1 combined with the fact that $\mathbf{Z}$ is a Diophantine subset of $R$ proves that the relation Par is Diophantine over $R[t]$. The relation Pos is Diophantine over $R[t]$ by Lemma 2.4 and condition (7) is Diophantine by Lemma 2.1.

The theorem now follows as described in the introduction.

\section{REMARKS}

Notice that the results in the first two sections are valid for a polynomial ring over the ring of integers in a real number field (provided that $\mathbf{Z}$ is Diophantine in this ring). We only use the fact that $K$ is a totally real field in the proof of the Main Lemma. How to generalise the result to polynomial rings over totally imaginary rings of integers seems a more difficult problem (compare with the difficulties of proving the undecidability of the Hilbert's Tenth Problem for $\mathbf{C}(t)$ in contrast to $\mathbf{R}(t)$; see [5] and also [12] ).

\section{REFERENCES}

1. Colliot-Thélène J.-L., Coray D., Jansuc J.-L. : Descente et principe de Hasse pour certaines variétés rationelles, Journal fur die Reine und Angewandte Mathematik 320 (1980), 150-191 MR 82f: 14020

2. Denef J.: Hilbert's Tenth Problem for quadratic rings, Proceedings of the American Mathematical Society, 48 (1975), 214-220 MR 50:12961

3. Denef J.: Diophantine sets over $\mathbf{Z}[t]$, Proceedings of the American Mathematical Society, 69 (1978), 148-150 MR 57:2899

4. Denef J.: Diophantine sets over algebraic integer rings 2, Transactions of the American Mathematical Society, 257 (1980), 227-236 MR 81b:12031

5. Denef J.: The diophantine problem for polynomial rings and fields of rational functions, Transactions of the American Mathematical Society, 242 (1978), 381-399 MR 58:10809 
6. Denef J.: The Diophantine Problem for polynomial rings in positive characteristic, Logic Colloqium '78, M. Boffa, D. van Dalen, K. McAloon (eds.),85-95, North Holland 1984 MR 81h:03090

7. Denef J., Lipshitz L.: Diophantine sets over some rings of algebraic integers, Journal of the London Mathematical Society (2), 18 (1978), 385-391 MR 80a:12030

8. Davis M., Putnam H.: Diophantine sets over polynomial rings, Illinois Journal of Mathematics, 7 (1963), 251-256 MR 26:4903

9. Matijasevich: Enumerable sets are diophantine, Doklady Akademii Nauka SSSR, 191 (1970); 272-282

10. Pourchet Y.: Sur la représentation en somme de carrés des polynômes a une indéterminé sur un corps de nombres algébriques, Acta Arithmetica, 19 (1971), 89-104 MR 44:6632

11. Pheidas T.: Hilbert's Tenth Problem for a class of rings of algebraic integers, Proceedings of the American Mathematical Society, 104 (1988), 611-620 MR 90b:12002

12. Pheidas, T.: Extensions of Hilbert's Tenth Problem, The Journal of Symbolic Logic, 59 (1994), nr.2, 372-397 MR 95f:03068

13. Rabin M.O.: Computable algebra, general theory and theory of computable fields, Transactions of the American Mathematical Society, 95 (1960), 341-360 MR 22:4639

14. Shlapentokh A.: Extensions of Hilbert's Tenth Problem to some algebraic number fields, Communications on Pure and Applied Mathematics, 42 (1989), nr.7, 939-962 MR 91g:11155

15. Shapiro H.,Shlapentokh A.: Diophantine relationships between algebraic number fields, Communications on Pure and Applied Mathematics, 42 (1989), nr.8, 1113-1122 MR 92b:11018

Universiteit Gent, Vakgroep Kwantitatieve Technieken, Hoveniersberg 4, B-9000 Gent, Belgium

E-mail address: Karim.Zahidi@rug.ac.be

Current address: Department of Applied Mathematics and Computer Science, Krijgslaan 281, sg, B-9000 Gent, Belgium 